gastrointestinal disease activity. ${ }^{2}$ Due to the relative rarity of these noncontiguous granulomatous lesions, there are no clinical trials to guide current treatment. Therapy with steroids, metronidazole, azathioprine, sulfasalazine, and tetracyclines has been used with variable success. ${ }^{2,3}$

Metastatic Crohn's disease exhibits variable clinical features and may resemble many other dermatoses. In this case of a female patient with active Crohn's and vulva and perineal ulcers other diagnoses such as Bechet's disease and a variety of sexually transmitted diseases were entertained. The diagnosis was ultimately made by biopsy of the patient's lesions. It is the recommendation of many experts in the field of inflammatory bowel disease that any unusual cutaneous lesion or any lesion that doesn't resolve with standard therapy be biopsied. ${ }^{2,3}$

\section{References}

1. Parks AG, Morson BC, Pegum JS. Crohn's disease with cutaneous involvement. Proc R Soc Med 1965;58:241-2.

2. Marotta PJ, Reynolds RPE. Metastatic Crohn's disease. Am J Gastroenterol 1996;91:373-5.

3. MacayaA, Marcoval J, BordasX, Morena A, Vazquez S, Peyri J. Crohn's disease presenting as prepuce and scrotal edema. J Am Acad Dermatol 2003;49:S182-3.

4. Guest GD, Fink RL. Metastatic Crohn's disease: case report of an unusual variant and review of the literature. Diseases of the Colon and Rectum 2000;43:1764-6.

\title{
INFILTRATED EPINEPHRINE
}

Utpal Sagar, MD, Bonnie Callahan, MD, and Ted Martynowicz, DO

\section{Case Presentation}

The patient is a 67 year-old male with past medical history of CAD s/p CABG, CHF with EF of $15 \%$, BiV- ICD, who was recently admitted to an outside hospital in Delaware with SOB and weakness, and was found to have frequent bouts of VT. During that hospitalization, he had suffered cardiac arrest as well, and epinephrine was administered emergently through a peripheral IV site located in his right hand. He was thereafter loaded with amiodarone for the ventricular tachycardia. It was later found that the epinephrine had infiltrated into the skin of his right hand from the peripheral IV site. Following stabilization at the outside hospital, the patient requested transfer to Wilmington VAMC for further treatment and rehabilitation. The photograph below (obtained with permission), illustrates the vast degree of skin necrosis and surrounding erythema that resulted from skin infiltration of epinephrine in this patient.

Vasoactive drugs are often administered immediately before, during, and after an arrest to support cardiac output. Drugs may be selected to improve chronotropy, inotropy, or arterial pressure. 1 The emergent nature of a cardiac arrest code often demands expedient administration of IV medications, at times through a peripheral IV site. This is done, however, with a certain amount of risk. In the event that extravasation develops, immediately infiltrate 5 to $10 \mathrm{mg}$ of phentolamine diluted in 10 to $15 \mathrm{~mL}$ of saline into the site of extravasation to prevent tissue necrosis, ${ }^{1}$ such as that observed in the above photograph.

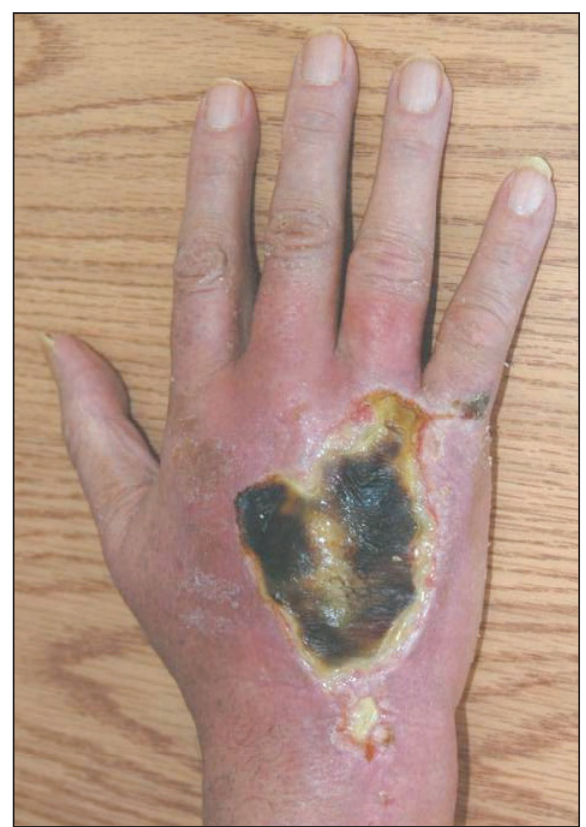

Photograph Courtesy of the Wilmington VA Medical Center.

\section{Reference}

1. 2005 American Heart Association Guidelines for Cardiopulmonary Resuscitation and Emergency Cardiovascular Care, Circulation. 2005;112:IV-78 - IV-83. 